\title{
A Product Quasi-Interpolation Method for Weakly Singular Volterra Integral Equations
}

\author{
Eero Vainikko and Gennadi Vainikko \\ Faculty of Mathematics and Computer Science, University of Tartu; J. Liivi 2, 50409 Tartu, Estonia
}

\begin{abstract}
For a weakly singular Volterra integral equation, we propose a method of Nyström type of accuracy $O\left(h^{m}\right)$ based on the smoothing change of variables and on the product quasi-interpolation by smooth splines of degree $m-1$ on the uniform grid.
\end{abstract}

Keywords: Volterra integral equation, weak singularities, spline quasi-interpolation, product integration, Nyström type methods.

PACS: $02.30 . \mathrm{Rz} ; 02.60 .-\mathrm{x}$

\section{INTRODUCTION}

Different methods of Nyström type for weakly singular Volterra and Fredholm integral equations have been constructed in [1], [2], [4]. In the present paper, we propose for a weakly singular Volterra integral equation a method of Nyström type of accuracy $O\left(h^{m}\right)$ based on the smoothing change of variables and on the product quasi-interpolation by smooth splines of degree $m-1$ on the uniform grid. Similar method for weakly singular Fredholm equations has been developed in [5].

\section{THE PROBLEM}

Consider the weakly singular Volterra integral equation

$$
u(x)=\int_{0}^{x}\left(a(x, y)(x-y)^{-v}+b(x, y)\right) u(y) d y+f(x), \quad 0 \leq x \leq 1,
$$

where $0<v<1, a$ and $b$ are defined and $C^{m}$-smooth for $0 \leq x \leq 1,0<y \leq x+\delta, \delta>0, m \in \mathbf{N}$, and satisfy there for $k+l \leq m$ the inequalities

$$
\left|\partial_{x}^{k} \partial_{y}^{l} a(x, y)\right| \leq c y^{-\lambda-l}, \quad\left|\partial_{x}^{k} \partial_{y}^{l} b(x, y)\right| \leq c y^{-\mu-l}, \quad v+\lambda<1, \quad \mu<1
$$

With the change of variables

$$
x=t^{r}, \quad y=s^{r}, \quad 0 \leq t \leq 1, \quad 0 \leq s \leq t+\delta_{r}, \quad r \in \mathbf{N}, \quad\left(1+\delta_{r}\right)^{r}=1+\delta,
$$

equation (1) takes with respect to $v(t)=u\left(t^{r}\right)$ the form

$$
v(t)=\int_{0}^{t}\left(\mathscr{A}(t, s)(t-s)^{-v}+\mathscr{B}(t, s)\right) v(s) d s+g(t), \quad 0 \leq t \leq 1,
$$

which is similar to (1). Here

$$
\begin{aligned}
& g(t)=f\left(t^{r}\right), \quad \mathscr{A}(t, s)=r a\left(t^{r}, s^{r}\right) \Phi(t, s)^{-v} s^{r-1}, \quad \mathscr{B}(t, s)=r b\left(t^{r}, s^{r}\right) s^{r-1}, \\
& \Phi(t, s)=\left\{\begin{array}{ll}
\frac{t^{r}-s^{r}}{t-s}, & t \neq s \\
r t^{r-1}, & t=s
\end{array}\right\}=\sum_{k=0}^{r-1} t^{r-1-k} s^{k}, \quad 0 \leq t \leq 1, \quad 0<s \leq t+\delta_{r} .
\end{aligned}
$$

We assume that the smoothing parameter $r \in \mathbf{N}$ satisfies the inequalities

$$
r>(1-v) /(1-v-\lambda), \quad r>1 /(1-\mu)
$$

Then $\mathscr{A}(t, s) \rightarrow 0, \mathscr{B}(t, s) \rightarrow 0$ as $s \rightarrow 0,0 \leq t \leq 1$. Extending $\mathscr{A}(t, s)$ and $\mathscr{B}(t, s)$ by the zero value for $s \leq 0$, the extended $\mathscr{A}(t, s)$ and $\mathscr{B}(t, s)$ are continuous for $0 \leq t \leq 1,-\infty<s \leq t+\delta_{r}$.

CP936, Numerical Analysis and Applied Mathematics, International Conference edited by T. E. Simos, co-edited by G. Psihoyios and Ch. Tsitouras

(C) 2007 American Institute of Physics 978-0-7354-0447-2/07/\$23.00 


\section{OPERATOR FORM OF THE METHOD}

Let $h=1 / n, n \in \mathbf{N}, n \geq(m-1) / \delta_{r}$. We call attention to a product quasi-interpolation method which we first present in the operator form:

$$
\begin{gathered}
v_{n}(t)=\int_{0}^{t}\left[(t-s)^{-v} Q_{h, m}^{\prime}\left(\mathscr{A}(t, s) v_{n}(s)\right)+Q_{h, m}^{\prime}\left(\mathscr{B}(t, s) v_{n}(s)\right)\right] d s+g(t), \quad 0<t<1, \\
v_{n}(t)=\left(\Lambda_{m} v_{n}\right)(t) \quad \text { for } 1 \leq t \leq 1+(m-1) h .
\end{gathered}
$$

Here $\Lambda_{m} v$ is the Lagrange interpolant of $v$ by polynomials of degree $m-1$ constructed using, in case of even $m$, the knots $1-j h, j=0, \ldots, m-1$, and in case of odd $m$, the knots $1-\left(j+\frac{1}{2}\right) h, j=0, \ldots, m-1$, whereas $Q_{h, m}^{\prime} w$ is the quasiinterpolant of $w$ by polynomial splines of degree $m-1 \geq 2$, defect 1 , with spline knots $j h, j \geq-m+1$ constructed in [3]. Namely, for a function $w(s), s \in[-(m-1) h,([n t\rceil+(m-1)) h]$, depending on $t, 0<t \leq 1$, as a parameter, the quasi-interpolant $Q_{h, m}^{\prime} w$ is defined for $s \in[0, t]$ by the formula

$$
\left(Q_{h, m}^{\prime} w\right)(s)=\sum_{j=-m+1}^{\lceil n t\rceil-1}\left(\sum_{|p| \leq m_{1}-1} \alpha_{p, m}^{\prime} w\left(\left(j-p+\frac{m}{2}\right) h\right)\right) B_{m}(n s-j),
$$

where $\lceil n t\rceil$ is the smallest integer $\geq n t$,

$$
\begin{gathered}
m_{1}=\left\{\begin{array}{cc}
\frac{m}{2}+1, & m \text { even } \\
\frac{m+1}{2}, & m \text { odd }
\end{array}\right\}=m-m_{0}, \quad m_{0}=\left\{\begin{array}{cc}
\frac{m}{2}-1, & m \text { even } \\
\frac{m-1}{2}, & m \text { odd }
\end{array}\right\}, \\
B_{m}(x)=\frac{1}{(m-1) !} \sum_{i=0}^{m}(-1)^{i}\left(\begin{array}{c}
m \\
i
\end{array}\right)(x-i)_{+}^{m-1}, \quad x \in \mathbf{R} \text {, is the father B-spline, } \\
\alpha_{p, m}^{\prime}=\sum_{q=|p|}^{m_{1}-1}(-1)^{k+q}\left(\begin{array}{c}
2 q \\
k+q
\end{array}\right) \gamma_{q, m}, \quad|p| \leq m_{1}-1, \\
\gamma_{0, m}=1, \quad \gamma_{q, m}=\sum_{l=1}^{m_{0}} \frac{\left(1+z_{l, m}\right) z_{l, m}^{m_{0}+q-1}}{\left(1-z_{l, m}\right)^{2 q+1} P_{m}^{\prime}\left(z_{l, m}\right)}, \quad q \geq 1,
\end{gathered}
$$

$z_{l, m} \in(-1,0), l=1, \ldots, m_{0}$, are roots of the characteristic polynomial $P_{m}(z)=\sum_{|k| \leq m_{0}} B_{m}\left(k+\frac{m}{2}\right) z^{k+m_{0}} \quad$ (they are simple; $1 / z_{l, m} \in(-\infty,-1), l=1, \ldots, m_{0}$, are the other $m_{0}$ roots of $\left.P_{m} \in \mathscr{P}_{2 m_{0}}\right)$.

\section{MATRIX FORM OF THE METHOD}

Note that $v_{n}(0)=g(0)=f(0)$. The solution $v_{n}$ of problem $(6)-(7)$ is uniquely determined on $[0,1]$ by the knot values $v_{n}\left(\left(i+\frac{m}{2}\right) h\right)$ for $0<\left(i+\frac{m}{2}\right) h \leq 1$. Collocating (6) at these points, the matrix form of method (6)-(7) follows. For even $m$, we obtain with respect to $v_{i, n}:=v_{n}(i h), i=1, \ldots, n+m$, the system of linear equations

$$
v_{i, n}=\sum_{k=1}^{i+m-1} \tau_{i, k} v_{k, n}+g(i h), \quad i=1, \ldots, n, \quad v_{i, n}=\sum_{j=0}^{m-1} \sigma_{i, j} v_{n-j, n}, \quad i=n+1, \ldots, n+m-1,
$$

where

$$
\begin{gathered}
\sigma_{i, j}=\prod_{j \neq j^{\prime}=0}^{m-1} \frac{j^{\prime}+(i-n)}{j^{\prime}-j}, \quad i=n+1, \ldots, n+m-1, \quad j=0, \ldots, m-1, \\
\tau_{i, k}=a_{i, k} \sum_{j=k-m}^{\min \{k, i-1\}} \beta_{i, j} \alpha_{j-k+m / 2, m}^{\prime}+b_{i, k} \sum_{j=k-m}^{\min \{k, i-1\}} \beta_{i, j}^{0} \alpha_{j-k+m / 2, m}^{\prime}, \quad i=1, \ldots, n, \quad k=1, \ldots, n+m-1, \\
a_{i, k}=\mathscr{A}(i h, k h), \quad b_{i, k}=\mathscr{B}(i h, k h), \quad, i=1, \ldots, n, \quad k=1, \ldots, n+m-1, \\
\beta_{i, j}=\int_{0}^{i h}(i h-s)^{-v} B_{m}(n s-j) d s, \quad \beta_{i, j}^{0}=\int_{0}^{i h} B_{m}(n s-j) d s, \quad i=1, \ldots, n, \quad j=-m+1, \ldots, i-1 .
\end{gathered}
$$

The unknowns $v_{i, n}, i=n+1, \ldots, n+m$, can be eliminated from system (8). 


\section{FORMULAE FOR QUADRATURE COEFFICIENTS (9)}

Again for even $m \geq 3$,

$$
\beta_{i, j}^{0}=\frac{h}{m !} \triangle^{m} \gamma_{i, j}^{0}, \quad \beta_{i, j}=h^{1-v} \triangle^{m} \gamma_{i, j}, \quad i=1, \ldots, n, \quad j=-m+1, \ldots, i-1,
$$

where $\triangle^{m}$ is the forward difference of order $m, \Delta \gamma_{i, j}=\gamma_{i, j+1}-\gamma_{i, j}$

$$
\begin{gathered}
\gamma_{i, j}^{0}=(j-i)^{m}-j^{m}, \quad \gamma_{i, j}=\sum_{k=0}^{m-1} \frac{(-1)^{m-k} i^{m-v-k}}{k !(1-v) \ldots(m-k-v)} j^{k} \quad \text { for } j=-m+1, \ldots,-1, \\
\gamma_{i, j}^{0}=(j-i)^{m}, \quad \gamma_{i, j}=\frac{(-1)^{m}}{(1-v) \ldots(m-v)}(i-j)^{m-v} \quad \text { for } 0 \leq j \leq i-1, \quad \gamma_{i, j}^{0}=\gamma_{i, j}=0 \quad \text { for } j \geq i .
\end{gathered}
$$

There are some symmetries for $\beta_{i, j}$ and $\beta_{i, j}^{0}$; it holds $\beta_{i, j}^{0}=h$ for $0 \leq j \leq i-m$.

\section{CONVERGENCE AND ERROR ESTIMATES}

Having solved system (8) we can use the Nyström extension to compute the solution $v_{n}(t)$ of problem (6)-(7) for all $t \in[0,1]$; a cheaper extension $\tilde{v}_{n}(t)$ can be constructed quasi-interpolating by splines of degree $m-1$ the solution of system (8) completed by $v_{i, n}=f(0)$ for $i=-m+1, \ldots,-1$. Introduce the space

$$
C_{\star}^{m}(0,1]=\left\{f \in C[0,1] \cap C^{m}(0,1]:\left|f^{(k)}(x)\right| \leq c_{f} x^{-k}, \quad 0<x \leq 1, \quad k=0, \ldots, m\right\} ;
$$

the smallest constant $c_{f}$ defines the norm $\|f\|_{C_{*}^{m}(0,1]}$.

\section{Theorem 1.}

(i) If $f \in C[0,1]$, the functions $a, b$ are continuous and satisfy (2) for $k=l=0$, and $r \in \mathbf{N}$ satisfies (5), then $\max _{0 \leq t \leq 1}\left|v(t)-v_{n}(t)\right| \rightarrow 0$ as $n \rightarrow \infty$ where $v$ and $v_{n}$ are the solutions of (4) and (6)-(7), respectively.

(ii) If $f \in C_{\star}^{m}(0,1]$, the functions $a, b$ are $C^{m}$-smooth for $0 \leq x \leq 1,0<y \leq x+\delta$ and satisfy (2) for $k+l \leq m$, and $r \in \mathbf{N}$ satisfies the inequatities $r>(m+v) /(1-\lambda), r>m /(1-\mu)$, then

$$
\delta_{m, n, r}:=\max _{0 \leq t \leq 1} t^{(r-1) v}\left|v(t)-v_{n}(t)\right| \leq c_{a, b, m, v, \lambda, \mu, r} h^{m}\|f\|_{C_{\star}^{m}(0,1)}
$$

(iii) Under the same conditions on $f, a, b$ as in (ii) but $r>m /(1-v-\lambda), r>m /(1-\mu)$, it holds

$$
\varepsilon_{m, n, r}:=\max _{0 \leq t \leq 1}\left|v(t)-v_{n}(t)\right| \leq c_{a, b, m, v, \lambda, \mu, r} h^{m}\|f\|_{C_{\star}^{m}(0,1)}
$$

Proof. The proof is based on the compact convergence of operators and on the error estimates of quasi-interpolation established in [3].

Remark 1. Claim (i) is true also for $\tilde{v}_{n}$; error estimates like in (i) and (ii) hold for $\tilde{v}_{n}$ under a slightly strengthened condition on $f \in C[0,1] \cap C^{m}(0,1]$.

Remark 2. If $f(0)=0$, the first condition on $r$ in (ii) and (iii) can be relaxed.

\section{SOME EXTENSIONS OF THE CONSIDERATIONS}

The results of Sections $2-6$ have been extended in the the following directions:

- in cases $m=1$ and $m=2$, the algorithms have a special treatment;

- in the case of odd $m \geq 3$, the algorithms are similar to those in Sections 4- 5;

- equations with logarithmic diagonal singularity of the kernel are treated;

- the case of $a$ and $b$ in (1) given only for $0 \leq s \leq t \leq 1$ is treated. 


\section{NUMERICAL TESTING}

Method (6) (7) and its modifications were tested numerically on the equation (1) with $v=1 / 2, a \equiv 1, b \equiv 0$,

$f(x)=1-x^{1 / 2}-\frac{\pi}{2} x$; the exact solution is then $u(x)=1+x^{1 / 2}$. About numerical results in the case of Fredholm equation, see [5].

\section{ACKNOWLEDGMENTS}

This work was supported by the Estonian Technology Foundation, grant No 05-03-01-35, and by the Estonian Science Foundation, grant No 5859.

\section{REFERENCES}

1. K. E. Atkinson, The Numerical Solution of Integral Equations of the Second Kind, Cambridge University Press, Cambridge, 1997.

2. H. Brumer, Collocation Methods for Volterra Integral and Related Functional Equations, Cambridge University Press, 2004.

3. E. Leetma and G. Vainikko, Math. Mod. Anal. 12, 107-120 (2007).

4. G. Monegato, and L. Scuderi, Math. Comput. 67, 1493 - 1515 (1998).

5. E. Vainikko and G. Vainikko, A product quasi-interpolation method for weakly singular Fredholm integral equations (submitted). 